\title{
HRJ
}

\author{
V.3 n.15 (2022)
}

Recebido: 18/10/2021

Aceito: 10/01/2022

\section{Análise da prescrição do fitoterápico Erva Baleeira (Cordia verbenacea DC) como recurso terapêutico no controle da dor}

\section{Raniere Pereira Gonçalves ${ }^{1}$ \\ Diego Era ${ }^{2}$ \\ Alexandre Vaz Machado ${ }^{3}$}

${ }^{1}$ Fisioterapeuta. Residente do Programa de Residência Multiprofissional em Saúde da família e comunidade, Escola Superior de Ciências da Saúde - ESCS/FEPECS. E-mail: ranifisio@ gmail.com.

${ }^{2}$ Fisioterapeuta. Pós-Graduando em Medicina Tradicional Chinesa - Acupuntura pela Universidade de BrasíliaUNB; Preceptor do Programa de Residência Multiprofissional em Saúde da Família e Comunidade da Escola Superior de Ciências da Saúde - ESCS/FEPECS. E-mail: diegoera@gmail.com

${ }^{3}$ Farmacêutico. Mestre em Ciências Farmacêuticas pela Universidade de Brasília-UNB (2018), Preceptor e Tutor do Programa de Residência Multiprofissional em Saúde da Família e Comunidade da Escola Superior de Ciências da Saúde - ESCS/FEPECS. E-mail: alexandrefarmunb@gmail.com

\section{RESUMO}

A fitoterapia é a ciência que estuda as plantas medicinais, suas finalidades e aplicabilidades na tríade de promoção, prevenção e reabilitação da saúde. A erva baleeira (Cordia verbenacea DC) é um arbusto perene, nativo do Brasil, utilizado no tratamento de diversos processos inflamatórios e infecciosos. Baseando-se no exposto, este trabalho reporta o uso do fitoterápico por pacientes da Atenção Primária à Saúde como recurso terapêutico à fisioterapia. $\mathrm{O}$ estudo teve como objetivo analisar a resposta à dor de pacientes que receberam a prescrição da erva baleeira em compressa. Foram entrevistadas nove pessoas de ambos os sexos, entre 49 a 72 anos, sendo a maioria do sexo feminino, em uma Unidade Básica de Saúde da cidade de Santa Maria - Distrito Federal. Foi aplicado um questionário pelo qual buscou-se obter o máximo de informações sobre o uso da compressa e seus benefícios. Para mensurar a dor, utilizou-se a Escala Visual Analógica (EVA) (Visual Analogue Scale - VAS): instrumento unidimensional, graduado de 0 a 10, para avaliação da intensidade da dor. A análise das respostas demonstrou redução e controle no quadro álgico de dor após uso da compressa, facilidade de manipulação do fitoterápico, aceitabilidade e boa resposta à necessidade de novas prescrições.

Palavras-chave: Fisioterapia; atenção primária à saúde; erva baleeira; fitoterapia; dor

Analysis of the prescription of herbal medicine Erva Baleeira (Cordia verbenacea DC) as a therapeutic resource for pain reduction.

\begin{abstract}
Phytotherapy is the science that studies medicinal plants, their purposes and applicability in the triad of health promotion, prevention and rehabilitation. The whaling herb (Cordia Verbenácea DC) is a perennial shrub, native to Brazil, used in several inflammatory and infectious processes. Based on the above, this work reports the use of herbal medicine by
\end{abstract}


patients in Primary Health Care as a therapeutic resource for physiotherapy. Objective: To analyze the response to pain of patients who received the prescription of erva baleeira in a compress. Health Center in the city of Santa Maria - Distrito Federal. A questionnaire was applied to obtain as much information as possible about the use of the compress and its benefits. To measure pain, the Visual Analogue Scale (VAS) was used (Visual analogue Scale-VAS): One-dimensional instrument to assess pain intensity, graded from 0 to 10 . Results: Response analysis showed reduction and control in the picture. pain after using the compress, ease of handling the herbal medicine, acceptability and good response to the need for new prescriptions.

Keywords: Physiotherapy; primary health care; Cordia verbenacea $D C$; phytotherapy, pain

\section{INTRODUÇÃO}

A Atenção Primária à Saúde (APS) é a porta de entrada do usuário ao Sistema Único de Saúde (SUS), tendo como papel principal ordenar as ações e serviços disponibilizados na Rede de Atenção à Saúde. Em 1993, no intuito de organizá-la, foi elaborado o Programa Saúde da Família. Já em 2006, houve mudanças e esse programa passou a ser chamado de Estratégia Saúde da Família (ESF). Desde então, tornou-se uma diretriz definitiva para reorganizar os serviços de saúde no Brasil, a fim de implantar os princípios e diretrizes do SUS e fortalecer a APS no país ${ }^{1}$.

A APS, através de suas equipes multidisciplinares, tem como objetivo trabalhar e evoluir a promoção da saúde, prevenção, recuperação e reabilitação de pessoas, reduzindo assim a demanda por serviços de atenção secundária e terciária²

Entre os desafios do SUS, estão: a expansão do acesso a todos, melhoria da qualidade dos serviços oferecidos e a resolução das ações na APS, diminuindo a necessidade do usuário em procurar outros níveis de atenção à saúde para solucionar o seu caso. A criação do Núcleo Ampliado de Saúde da Família (NASF) vem com esse propósito de ampliar o escopo e a cobertura, reforçando a implantação da resolutividade, solucionando problemas antes direcionados aos demais níveis de atenção à saúde ao reduzir custos e demanda dos hospitais³. 
Dentre uma das formas de colaborar com a resolução de algumas demandas e desafios apresentados dentro dos serviços da APS, está a Fitoterapia, um vasto recurso terapêutico que tem sido bastante explorado nos serviços de saúde.

A Fitoterapia é a ciência que estuda as plantas medicinais, suas finalidades e aplicabilidades na tríade de promoção, prevenção e reabilitação da saúde. Já o fitoterápico define-se como um produto com ações medicinais adquirido da planta ou de seus compostos, exceto substâncias isoladas, com finalidade curativa, preventiva ou paliativa ${ }^{4}$.

No Distrito Federal (DF), somente em 2014 foi instituída a Política Distrital de Práticas Integrativas do Distrito Federal (PDPIS). Porém, as práticas já são oferecidas e ofertadas pelo DF no âmbito do SUS há pelo menos 30 anos, segundo registros apontados na própria lei distrital. Desde 1989, a Fitoterapia é uma das práticas integrativas oferecidas pelo SUS no DF em atendimentos médico-ambulatoriais, juntamente como a Acupuntura, Homeopatia e a Alimentação Natural. Mais tarde, em 1998, inaugurou-se no DF o laboratório de Manipulação de Medicamentos Fitoterápicos e Homeopáticos ${ }^{5}$.

O profissional fisioterapeuta que atua na APS, assim como nos demais níveis de atenção, está apto a prescrever fitoterápicos, segundo a resolução do Conselho Federal de Fisioterapia e Terapia Ocupacional (COFFITO). O Acórdão n $^{\circ} 611$, de $1^{\text {o }}$ de abril de 2017, expressa que o fisioterapeuta poderá adotar a prescrição de fitoterápicos relacionados ao seu campo de atuação. Nesse cenário, o COFFITO visa aperfeiçoar a utilização e/ou indicação de substâncias de livre prescrição pelo fisioterapeuta, considerando o atual contexto científico e social para correto emprego das plantas medicinais e medicamentos fitoterápicos/fitofármacos, medicamentos antroposóficos, medicamentos homeopáticos, medicamentos ortomoleculares, florais, medicamentos de venda livre para fonoforese e iontoforese ${ }^{6}$. 
Dentro da Fitoterapia, a Cordia verbenacea DC, popularmente chamada de erva baleeira é um arbusto perene, de até dois metros de altura, nativo do Brasil e encontrado em todo o litoral brasileiro, principalmente em Santa Catarina. A erva baleeira, também conhecida como maria-preta, maria-milagrosa, catinga de barão ou pimenteira, é utilizada no tratamento da úlcera gástrica, artrite reumatóide e diversos processos inflamatórios e infecciosos ${ }^{7}$

Segundo a $2^{\circ}$ edição do Formulário de Fitoterápicos - Farmacopeia Brasileira (Agência Nacional de Vigilância Sanitária - ANVISA), o uso da erva baleeira tem uma contraindicação relativa, relacionada à ausência de informações e estudos adequados que comprovem a segurança de seu uso em grupos específicos, como gestantes, lactantes e pessoas com idade inferior a 18 anos. Recomenda-se o seu uso somente na pele íntegra. Em alguns casos raros, é possível haver hipersensibilidade ou reação alérgica na pele no local do uso da erva. Nessas ocorrências, deve-se suspender o uso da erva imediatamente e procurar atendimento médico ${ }^{8}$.

O presente trabalho torna-se relevante em razão do alto índice de pacientes da fisioterapia com dores musculotendíneas dentro da APS e os escassos recursos terapêuticos disponíveis nas UBS para tratamento dessas algias, considerando as compressas de erva baleeira um recurso terapêutico adicional e coadjuvante no tratamento desses pacientes. Além de ser uma forma adicional de complementação ao tratamento e com baixo custo, com menores efeitos adversos e boa aceitação, o uso da Fitoterapia é uma prática cultural bastante enraizada no Brasil, fator que facilita a adesão a esse tipo de tratamento.

\section{METODOLOGIA}

Trata-se de um estudo qualitativo e avaliativo baseado em entrevistas. As entrevistas, realizadas via teleatendimento/teleconsulta, utilizaram questionários semi estruturados, 
realizados por elaboração própria, como instrumento de coleta de dados, contendo perguntas divididas em dois segmentos a fim de facilitar a leitura do instrumento de coleta e análise dos resultados: dados clínicos (diagnóstico clínico, região do corpo humano que se localiza a dor, presença de sinais flogísticos, graduação da dor antes e depois do uso da compressa) e dados avaliativos quanto ao fitoterápico erva baleeira na forma de compressa (frequência de uso da compressa, quantidade de sachês utilizados, grau de dificuldade de aplicação da compressa).

O público-alvo foi composto por pacientes que foram atendidos pela equipe de Fisioterapia do NASF da UBS de Santa Maria - Distrito Federal, no período de abril a junho de 2021, para os quais foi prescrita a compressa da erva baleeira apenas por fisioterapeutas da unidade como um recurso terapêutico complementar à fisioterapia motora (cinesioterapia). Foram excluídos da pesquisa todos os pacientes que receberam a prescrição da compressa de erva baleeira por outros profissionais de saúde da UBS; pacientes que fizeram fisioterapia sem prescrição de compressa do fitoterápico; e aqueles que apesar de associarem a compressa e a fisioterapia em seu tratamento, estavam fora do período de coleta e análise dos dados da pesquisa.

Os participantes receberam uma prescrição de compressa desse fitoterápico, que fora produzido pela Farmácia Viva do DF - lote: 01/2021DVEB, acondicionado em sachê de trinta gramas cada, contendo o fármaco na forma de folhas trituradas, de uso tópico, de acordo com indicação contida também na embalagem, dispensado no mesmo dia pela farmácia da própria UBS. A orientação de preparo foi a infusão, adicionando uma colher de sopa do composto fitoterápico (extrato seco) em $150 \mathrm{~mL}$ de água fervente, devendo-se mexer bem e aguardar por cinco minutos. Após decorrido o tempo de descanso, recomenda-se filtrar o infuso e reservá-lo em recipiente de vidro. Em seguida, prepara-se a compressa com o auxílio de um algodão, pano ou gaze e aplica-se na região acometida, podendo ser utilizada em até três vezes ao dia ${ }^{9}$. 
A média de amostras coletadas na aplicação do questionário foi definida de acordo com o número de prescrições feitas por fisioterapeutas; número de atendimentos realizados pelos fisioterapeutas e número de fitoterápicos liberados pela Farmácia Viva do DF. Um total de nove participantes aceitou colaborar com o estudo. O número reduzido da amostra geral foi devido a inúmeros fatores: pandemia da Covid-19, nos quais todos os profissionais do NASF e da UBS estavam direcionados para a vacinação de combate a essa doença e à gripe. A Farmácia Viva também restringiu o número de compressas de ervas baleeiras produzidas e encaminhadas à UBS, onde foram coletados os dados. A justificativa da redução da produção e liberação do fitoterápico é o fato da pandemia do Coronavírus, da redução da produção devido ao déficit de mão de obra qualificada para a sua execução, além do problema com maquinário da farmácia.

Para mensurar a dor musculotendínea dos pacientes avaliados na pesquisa, utilizou-se a Escala Visual Analógica (EVA) para dor (Visual Analogue Scale - VAS): instrumento unidimensional para avaliação da intensidade da dor. Refere-se a uma linha com as extremidades numeradas de $0-10$, no qual 0 equivale a nenhuma dor e 10 significa uma dor insuportável. A avaliação quanto à dor foi questionada antes e após o uso da compressa de erva baleeira para posterior análise dos dados.

Para análise, foi construído um banco informatizado para armazenamento e leitura dos dados no formato de Excel® a fim de facilitar a coleta e a compilação dos dados referentes às variáveis: sexo; idade; utilização da compressa; escala de dor antes e após o uso da compressa de erva baleeira; facilidade e aplicabilidade da compressa e o tempo de uso desse fitoterápico oferecido nessa apresentação. Na coleta de dados, cada questionário recebeu uma identificação por meio de um número para facilitar a coleta de dados, não sendo uma forma de identificação do usuário, mantendo-se assim o sigilo e a não identificação do entrevistado. A 
descrição das variáveis foi dividida entre classes e analisada de acordo com a média e a porcentagem que aparece no estudo.

A pesquisa foi realizada mantendo sob sigilo todos os dados coletados e utilizados, de modo a garantir a proteção de todas as pessoas e entidades envolvidas, impedindo uma vinculação com qualquer profissional e/ou paciente específico, assegurando sua confidencialidade.

A pesquisa foi pautada pela Lei Geral de Proteção de Dados Pessoais - Lei $\mathrm{n}^{\circ}$ 13.709, de 14 de agosto de 2018. O artigo $1^{\circ}$ desta Lei dispõe sobre o tratamento de dados pessoais, inclusive nos meios digitais ${ }^{11}$. O Termo de Consentimento Livre e Esclarecido (TCLE) foi disponibilizado e realizado conforme legislação vigente e exigências do Comitê de Ética e Pesquisa (CEP). O projeto de pesquisa foi aceito pelo $\mathrm{CEP}$ sob o número: CAE 46605821.8.0000.5553 versão 2.

\section{RESULTADOS E DISCUSSÕES}

Os medicamentos fitoterápicos podem se apresentar de diversas formas: desde os tradicionais e mais difundidos pela cultura como os chás, até os mais elaborados e parecidos com os medicamentos sintéticos como comprimidos e cápsulas, além de gel, compressas e pomadas. A elaboração da forma farmacêutica vai depender de uma sucessão de requisitos que determinarão para qual tipo de forma de apresentação esse fitoterápico vai ser mais aceito e melhor aproveitado suas propriedades terapêuticas e curativas ${ }^{12}$.

No DF, o Núcleo de Farmácia Viva (NURFAV-DF) produz medicamentos fitoterápicos há anos. Entre eles, o fitoterápico de erva baleeira (Cordia verbenacea DC) é produzido e distribuído às farmácias das UBS da Secretaria de Estado e Saúde do Distrito Federal (SES-DF) em formas de gel, onde sua aplicação e utilização por parte dos profissionais que a prescrevem e os pacientes que utilizam o medicamento é muito bem aceita. Como alternativa para o tratamento de pacientes que necessitavam do fitoterápico com 
ação anti-inflamatória, optou-se por produzir a compressa de erva baleeira. Diante do exposto, surgiu a questão da avaliação do uso desse novo fitoterápico na apresentação de compressa, avaliando questões como a adesão da população a essa nova forma de apresentação do medicamento e a necessidade e carência de recursos terapêuticos para a área de Fisioterapia nas UBS.

O setor de Fisioterapia não conta com muitos recursos terapêuticos disponíveis. Em se tratando de equipamentos, principalmente para se trabalhar a dor e o processo inflamatório, o setor dispõe apenas de alguns equipamentos de cinesioterapia (halteres, caneleiras, faixas e bola macia) e orientações domiciliares, além de alguns recursos terapêuticos manuais próprios, a depender da formação do profissional.

A cinesioterapia se autodenomina, sendo a cinesio referente ao movimento e a terapia refere-se a tratamento, fundindo-se e formando o conceito do recurso, ou seja, o tratamento através do movimento do corpo e do exercício propriamente dito. Por intermédio dessa técnica, é possibilitado que o paciente alcance a prevenção, reabilitação e cura de suas patologias físicas $^{13}$.

Encontram-se na literatura várias modalidades de recursos terapêuticos utilizados na prevenção e no tratamento para a dor muscular com efeitos analgésicos e anti-inflamatórios. Entre os mais tradicionais, podem-se citar a termoterapia (uso do calor, como o ultrassom terapêutico); a crioterapia (uso do gelo); a eletroterapia (correntes elétricas); a fototerapia (uso do Laser); os exercícios terapêuticos (cinesioterapia) e as massagens terapêuticas ou recurso terapêutico manual (liberação miofascial) ${ }^{14}$.

Este trabalho foi realizado por meio de entrevista com nove pacientes, de ambos os sexos, sendo $66,67 \%$ do sexo feminino e $33,33 \%$ do sexo masculino com idades entre 52 e 72 anos com uma idade média de 59 anos entre os participantes, apresentando patologias musculotendíneas e dores musculares crônicas e agudas. 
As patologias mais predominantes que são comumente tratadas pela Fisioterapia Traumato-Ortopédica são: lombalgia; dorsopatias; escoliose; artrite reumatóide; artrose, tendinites; bursites; luxações e distensões musculares; fraturas; lesões por esforço repetitivo (LER), cervicalgia; dor ciática; hérnia de disco; entorse e osteoporose ${ }^{15,16}$. As patologias mais prevalentes no estudo estão representadas na figura 1 .

Figura 1. Dados clínicos extraídos do estudo de acordo com o diagnóstico clínico do paciente (\% Porcentagem).

\section{PRINCIPAIS PATOLOGIAS}

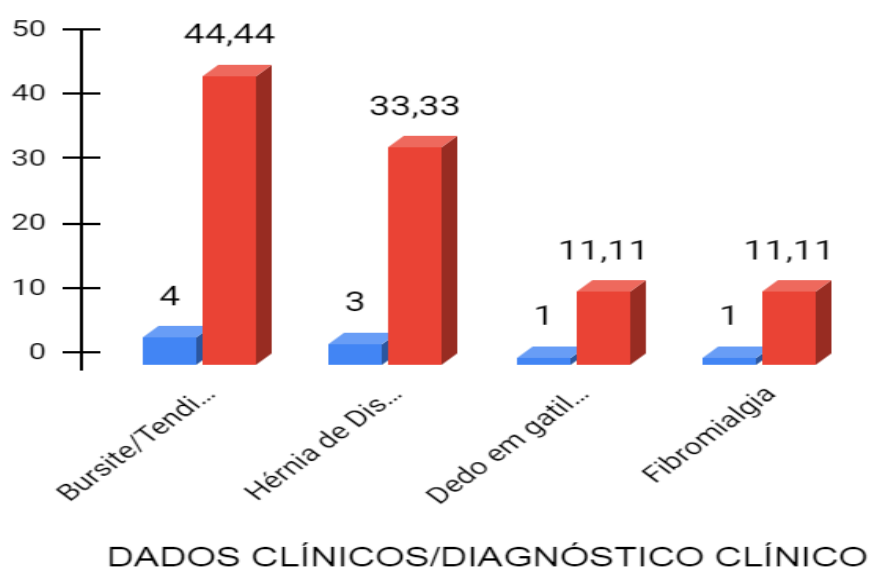

Fonte: dados da pesquisa / GONÇALVES 2021

Segundo os estudos citados no parágrafo acima e os resultados obtidos na entrevista, as patologias mais prevalentes na pesquisa atual são a bursite/tendinite e a hérnia de disco, resultando mais de $77 \%$ do total apresentado, semelhante ao encontrado em estudo realizado por Nogueira $(2016)^{15}$ e Nascimento $(2020)^{16}$. Quase todas que apareceram no estudo são prevalentes na Ortopedia e aparecem também frequentemente no consultório da APS. Na sequência, os dados obtidos indicam que o segmento corporal com maior acometimento entre 
os entrevistados é o ombro com bursites e tendinites, equiparado com a coluna com hérnia de disco. Essas enfermidades podem causar dor e limitações, gerando aos pacientes o afastamento de atividades laborais e sociais. A figura 2 apresenta os dados extraídos do questionário sobre essa variável.

Figura 2. Segmento corporal comprometido com maior persistência/ocorrência de dor (\% Porcentagem).

\section{SEGMENTO CORPORAL COMPROMETIDO}

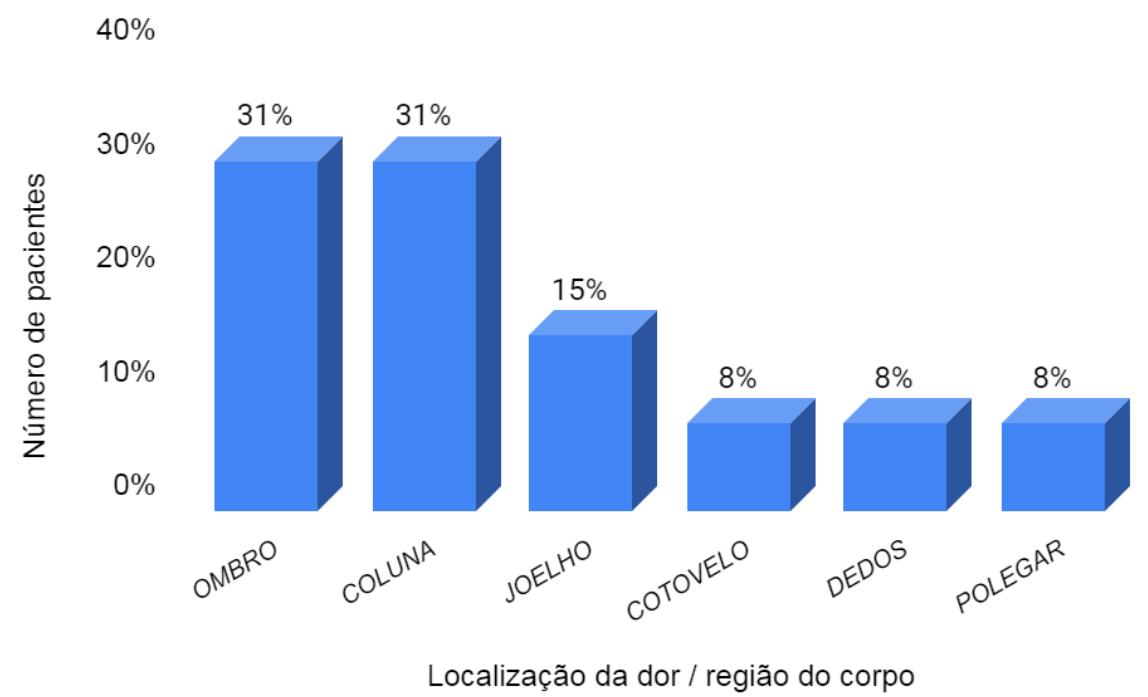

Fonte: dados da pesquisa / GONÇALVES, 2021

A avaliação de dor realizada durante as teleconsultas e entrevistas foi indicada na Escala Visual Analógica - EVA de dor e autodeclarada pelo entrevistado. Foi questionado o nível de dor antes e após a fisioterapia com a utilização da compressa de erva baleeira. Observa-se a redução da média de dor entre os entrevistados após a utilização do fitoterápico em forma de compressa associada à fisioterapia. Os dados a respeito da média de dor avaliada encontram-se na figura 3. 
Figura 3. Média de dor avaliada em questionário, segundo a EVA (Escala Visual Analógica) Graduados de 0 a 10 . Onde 0 é nenhuma dor e 10 é uma dor insuportável (Média aritmética).

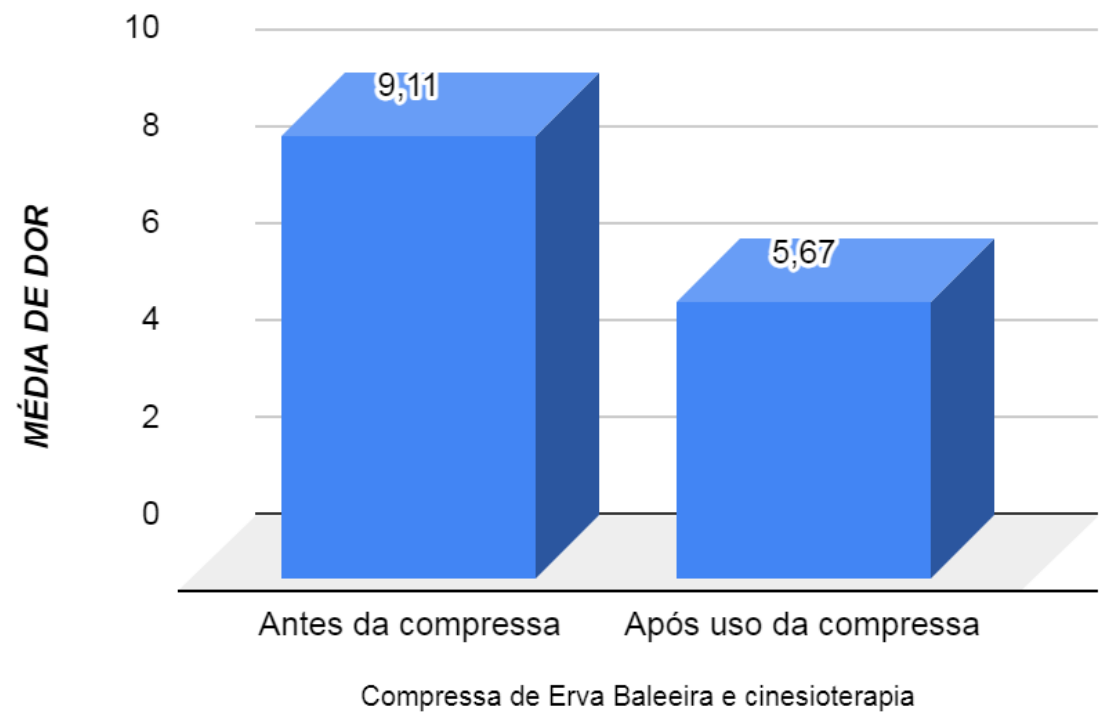

Fonte: dados da pesquisa / GONÇALVES 2021

A dor é uma sensação ou experiência subjetiva, personalíssima e intransferível, abrangendo fatores sensoriais, culturais e emocionais, modificados ou não por fatores socioculturais, psicológicos e fenômenos emocionais ${ }^{17}$.

A dor pode se dividir em crônica e aguda, dependendo de fatores como tempo, frequência; intensidade e outros. No estudo, atentou-se apenas na classificação de dor em crônica ou aguda, uma variável categórica utilizada no questionário referente à persistência de dor declarada pelo entrevistado.

A dor crônica pode ser determinada como dor constante e ininterrupta ou recorrente de durabilidade mínima de três meses, sendo definida por algumas literaturas a partir de seis meses de duração. Sua atribuição é de alerta e, geralmente, sua origem é indefinida, tendo uma eliminação de difícil manejo terapêutico convencional, sendo, portanto, motivo de incapacidades, limitações e restrições prolongadas ${ }^{18}$. 
Por outro lado, a dor aguda é considerada um distúrbio fisiológico, um indicador de que algo não está bem, um alerta emitido pelo nosso corpo de grande relevância para a sobrevivência da nossa espécie. Sua duração é limitada em relação ao período de tempo e espaço, geralmente se encerra quando a causa que a desencadeia é solucionada ${ }^{19}$. Segundo a sua origem, a grande maioria dos estudos relacionados à análise da dor aguda conclui que ela se manifesta após processos pós-operatórios, traumas em geral e queimaduras ${ }^{20,21}$.

A figura 4 apresenta os dados em relação a essa temática. O tipo de dor foi um fator relevante para o trabalho, pois o fitoterápico avaliado nesse estudo é utilizado nos processos inflamatórios que geralmente estão associados à presença de dor aguda. No entanto, o estudo também visa saber se há efeito satisfatório em usuários com dor crônica.

Figura 4: Percentual de acordo com o tipo de dor apresentado na entrevista, dor crônica ou dor aguda. Foi considerada dor crônica aquela presente por mais de 03 meses ( 90 dias), e dor aguda menor que este período (\% Porcentagem).

\section{TIPO DE DOR}

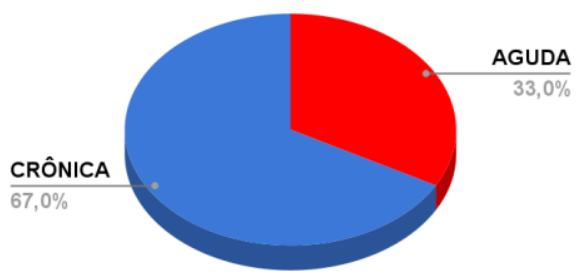

Fonte: dados da pesquisa / GONÇALVES, 2021

Nota-se que o número de pacientes com dor crônica é superior ao de dor aguda, mas, mesmo assim, a média de dor apresentada após a utilização do fitoterápico é satisfatória, pois mesmo em dores crônicas obteve-se a redução significativa da dor em geral.

Analisou-se a média de tempo de dor entre os participantes na qual a dor crônica foi calculada em relação aos meses de dor, enquanto a dor aguda foi calculada em média de dias. Os participantes do estudo apresentaram a seguinte média de tempo de dor: relato de dor crônica por 70 meses e relato de dor aguda por 57 dias. 
A inflamação é um instrumento de proteção local, um meio de defesa específico de alguns tecidos (tecido conjuntivo, ósseo, muscular, cartilaginoso, além de vasos sanguíneos e linfáticos). A inflamação é a resposta local do tecido irrigado o qual foi lesionado, definida por mudanças do sistema vascular, dos elementos líquidos e celulares, também por adequações do tecido conjuntivo adjacente. Existem dentro do processo inflamatório cinco sinais conhecidos como sinais cardeais ou sinais flogísticos. A ausência de alguns deles ou a sua presença isolada não justifica que seja manifestação de um processo inflamatório. São eles: edema; calor; rubor; dor e perda da função ${ }^{22}$.

Nos dados coletados durante o estudo, foi abordada a questão da presença de sinais cardeais ou sinais flogísticos antes da fisioterapia e da compressa de erva baleeira. Antes da coleta de dados, foi explicado de forma simples e popular o que cada sinal representa para ser um conhecimento homogêneo do dado antes de ser mensurado, dando mais fidelidade à resposta individual.

A presença de sinais flogísticos no estudo aparece com uma representação de $66,7 \%$ do total de pacientes, ou seja, esses pacientes apresentaram dois ou mais sinais que podem sugerir a presença de um processo inflamatório local. Apresentaram patologias que ocasionam processos inflamatórios, com bursites e tendinites. Dessa maneira, esses participantes estão mais propícios a já terem um processo inflamatório em curso ou estabelecido. Portanto, o uso da erva baleeira em compressa seria uma boa indicação em virtude de sua ação antiinflamatória e analgésica, tornando-se opção de complemento à fisioterapia no controle da dor.

A forma e os meios de utilização do fitoterápico supracitado foram analisados em questões agrupadas referentes ao tema em questão. Foi avaliada a forma da compressa, o meio de aplicação, utilização ou não da compressa prescrita, uso de fitoterápicos pelos 
entrevistados para outros meios de tratamento, a quantidade de receitas prescritas e também aceitabilidade/adesão da compressa e possibilidade de reuso desse medicamento.

Conforme representado na tabela 1 , as respostas foram altamente satisfatórias. $\mathrm{O}$ grupo estudado apresentou um percentual elevado em todos os quesitos averiguados, acima de $75 \%$ de aceitabilidade e facilidade para usar e manusear a compressa. Além de estar aberto a novas experiências sensoriais, houve a satisfação do resultado pelo paciente, implicando em aceitabilidade de utilizar em novos momentos caso necessário, mesmo sendo poucos os que tinham utilizado anteriormente algum medicamento fitoterápico.

Tabela 1: Relata os dados avaliados com os entrevistados quanto ao percentual \% da utilização do fitoterápico erva baleeira (cordia verbenanea DC) e se já utilizou algum outro fitoterápico.

\begin{tabular}{|c|c|c|c|c|}
\hline DADOS & SIM & NÃo & \% Maior número & $\%$ Menor número \\
\hline Utilizou a compressa recebida & 9 & 0 & $100 \%$ & \\
\hline Utiliza ou já utilizou fitoterápicos & 2 & 7 & $77,78 \%$ & $22,22 \%$ \\
\hline Foi precrito mais de uma receita & 0 & 9 & $100 \%$ & \\
\hline Utilizaria novamente & 8 & 1 & $88,89 \%$ & $11,11 \%$ \\
\hline
\end{tabular}

Fonte: dados da pesquisa / CONÇALVES 2021

Houve a análise da durabilidade de tempo do tratamento com a utilização do fitoterápico em conjunto com a fisioterapia e também da quantidade de fármaco prescrita e administrada pelos entrevistados. Para esses tópicos, foram realizadas perguntas direcionadas a quantos dias o medicamento foi administrado e quantos sachês foram usados, mesmo sabendo-se que cada paciente recebeu um sachê de trinta gramas prescrito e entregue pelo profissional Fisioterapeuta da unidade. As respostas dos dados seguem representadas na tabela 2. 
Tabela 2: Dados avaliados no estudo quanto ao uso (dias) e a quantidade de sachês de trinta gramas do fitoterápico erva baleeira (cordia verbenacea DC) (\% porcentagem).

\begin{tabular}{ccccc}
\hline \multicolumn{7}{c}{ DADOS / quantidade de sachês (30 gramas) } \\
\hline Utilizou quantos dias & $\mathbf{1}$ a $\mathbf{2}$ & $\mathbf{3}$ a 4 & $\mathbf{5}$ a $\mathbf{6}$ & $\%$ do maior número \\
Utilizou quantos saches & 0 & 2 & 7 & $77,78 \%$ \\
& 9 & 0 & 0 & $100 \%$ \\
\hline
\end{tabular}

Fonte: dados da pesquisa / GONÇALVES 2021

A maioria dos entrevistados $(77,8 \%)$ utilizou a compressa entre cinco e seis dias, a previsão ficaria em três dias se tivessem utilizado duas vezes ao dia, mas a maioria confessou ter utilizado apenas uma vez ao dia, geralmente no final da tarde ou à noite.

Foi questionada a quantidade utilizada nos preparos da compressa. Todos informaram com unanimidade que realizaram as compressas com uma colher de chá do preparado da erva baleeira em $150 \mathrm{~mL}$ de água filtrada. Em teleconsulta, relataram que após colocar a infusão na compressa, toalha ou pano, fizeram a utilização de um microondas, quem dispunha do equipamento, ou do ferro de passar roupas para aquecer um pouco a compressa. Desfrutando, dessa forma, de mais um recurso terapêutico colaborador da analgesia e redução do processo inflamatório, a termoterapia.

A termoterapia é determinada pela utilização do calor em forma terapêutica, ou seja, qualquer elemento que possa promover e aumentar a temperatura dos tecidos. Existe uma diversidade de meios e equipamentos para se obter os efeitos termoterapêuticos, podendo ser classificados de acordo com a sua profundidade de alcance de atuação nos tecidos, seja superficial ou profunda, e ainda sobre o seu meio de transferência de calor, seja por condução, convecção e conversão ${ }^{23,24}$.

Além das perguntas executadas na teleconsulta, realizadas pela profissional fisioterapeuta responsável pela coleta de dados, foram feitas orientações quanto aos exercícios fisioterapêuticos prescritos, inclusive quanto a sua continuidade e manutenção, exercícios 
físicos frequentes, além de tirar dúvidas pertinentes a cada paciente, exercendo a pesquisadora uma escuta qualificada. A entrevista/teleconsulta durou em média de 30 a 40 minutos cada e foi realizada após dois meses da prescrição e dispensação do fitoterápico.

\section{CONSIDERAÇÕES FINAIS}

Os resultados demonstram que a maioria dos entrevistados foram mulheres adultas com dores crônicas e com média de dor prolongada em relação a constante tempo. Elas apresentam sinais flogísticos, com o ombro e a coluna sendo o segmento corporal de maior comprometimento em relação a dor. A bursite/tendinite é a patologia mais prevalente, seguida da hérnia discal não especificada. As utentes afirmam que a compressa do fitoterápico erva baleeira (Cordia Verbenacea DC) teve resultado efetivo no controle de dor e no processo inflamatório, com redução da média de dor avaliada, não apenas em dores agudas, mas também nas consideradas cronificadas, mesmo diante de uso restrito de apenas um sachê de trinta gramas e por apenas 5 a 6 dias de uso da maioria, uma única vez ao dia.

Além de apresentar adequada aceitação do fitoterápico em compressa, mesmo diante de usuários não habituados a esse tipo de forma farmacêutica, houve também resultados satisfatórios quanto ao manejo e preparo do produto e adequado uso, além de abertura para aceitação de novas administrações, se necessário, sem restrição de dificuldade ou de desagrado da conduta.

O estudo trouxe mais um recurso a ser explorado pela comunidade multiprofissional de saúde - o uso de erva baleeira em compressa - principalmente aos fisioterapeutas, que podem utilizar do recurso para complementar a sua técnica de tratamento, obtendo um melhor resultado no manejo e controle de dor em processos musculares e tendinosos. Na APS, onde os recursos terapêuticos são escassos, essa pode ser uma alternativa de muito sucesso. 
Também é direcionado a profissionais fisioterapeutas e outros da APS que possam tomar como referência para prescrever o fitoterápico na forma de compressa. A literatura é muito escassa ainda em artigos e trabalhos direcionados para o tema em questão, logo, este estudo pode ser utilizado futuramente para políticas públicas de ampliação de Farmácias Vivas e distribuição de mais fitoterápicos em nível nacional.

Sugerem-se mais estudos, de preferência, com amostras mais significativas, já que o atual contou com uma amostra reduzida e análise de poucas variáveis devido às inúmeras limitações que este estudo enfrentou, como a pandemia de Covid-19, atendimentos individuais restritos e pontuais, suspensão de atendimentos em grupos, limitação de material para análise, remanejamento de servidores e residentes para apoiar a vacinação, redistribuição dos Fisioterapeutas do NASF para atendimentos específicos a usuários com demandas respiratórias, como para a sala vermelha da UBS, sala de acolhimento e atendimento do sintomático respiratório, dentre outras. Como sugestão, uma análise com outras faixas etárias, ou com atletas que sofrem muito com dores musculares, já que estes grupos não foram contemplados com o estudo atual, bem como um estudo de comparação entre o uso da compressa de erva baleeira e o gel do mesmo fitoterápico.

\section{REFERÊNCIAS}

1. FERRETTI, F. et al. Inserção de fisioterapeutas na equipe da Estratégia Saúde da Família: a visão dos usuários. Fisioter. Mov. Curitiba, v. 28, n. 3,p. 485-493, setembro de 2015. Disponível em: $\quad$ http://www.scielo.br/scielo.php?script=sci_arttext\&pid=S0103 51502015000300485\&lng=en\&nrm=iso. Acesso em: 01 ago. 2020. https://doi.org/10.1590/0103-5150.028.003.AO07.

2. SILVA, G. G. da; SIRENA, S. A. Perfil de encaminhamentos à fisioterapia por um serviço de Atenção Primária à Saúde, 2012. Epidemiol. Serv. Saúde, Brasília, v. 24, n. 1, p. 
123-133,Mar.

em:

http://www.scielo.br/scielo.php?script=sci_arttext\&pid=S2237-

96222015000100123\&lng=en\&nrm=iso. Acesso em: 01 ago. 2020. https://doi.org/10.5123/S1679-49742015000100014.

3. SOUZA, M. de O.; SANTOS, K. O. B. dos. Atuação do fisioterapeuta no Centro de Apoio à Saúde da Família. Fisioter. mov. Curitiba, v. 30, n. 2, pág.237-246, abril de 2017. Disponível em:

http://www.scielo.br/scielo.php?script=sci_arttext\&pid=S0103-

51502017000200237\&lng=en\&nrm=iso. Acesso em: $14 \quad$ set. 2020 . https://doi.org/10.1590/1980-5918.030.002.ao04 .

4. GOUVEIA, G. D. A.; SIMIONATO, C. Plantas medicinais e fitoterapia na atenção básica. Universidade Federal de Santa Catarina, Núcleo Telessaúde Santa Catarina. Florianópolis - SC. UFSC, 2019. Governo Federal. Disponível em: https://ares.unasus.gov.br/acervo/html/ARES/14863/1/Apostila_Fitoterapia_N\%C3\%BAcleo \%20Telessaude\%20SC\%20UFSC.pdf. Acesso em: 14 fev. 2021.

5. DISTRITO FEDERAL. Secretaria de Estado de Saúde do Distrito Federal -Subsecretaria de Atenção Primária à Saúde. Política Distrital de Práticas Integrativas em Saúde - Pdpis. Gerência de Práticas Integrativas em Saúde - Brasília: Distrito Federal- DF, FEPECS 2014. Disponível em: https://www.fcm.unicamp.br/fcm/sites/default/files/2016/page/pdpic_distrito_federal.pdf. Acesso em: 19 jun.2021.

6. BRASIL, ACÓRDÃO $N^{\circ} 611, D E 1^{\circ} D E$ ABRIL DE 2017. DIÁRIO OFICIAL DA UNIÃO. Publicado em:25/04/2017 | Edição: 78 | Seção: 1 | Página: 82.Órgão: Entidades de Fiscalização do Exercício das Profissões Liberais/CONSELHO FEDERAL DE FISIOTERAPIA E TERAPIA OCUPACIONAL. Disponível em 
https://www.in.gov.br/materia/-/asset_publisher/Kujrw0TZC2Mb/content/id/20178599/do12017-04-25-acordao-n-611-de-1-de-abril-de-2017-20178548. Acesso em: 14 fev. 2021.

7. JAYME A. A. SERTIÉ, A. C. B., PANIZZA S., MATIDA A. K. ZELNIK, R. Monografia Cordia verbenacea -Bulário- Acheflan ${ }^{\circledR}$ - Anti-inflammatory activity and sub-acute toxicity of Artemetin Revista Planta Médica, 1990. Disponível em: https://www.ppmac.org/sites/default/files/erva_baleeira.pdf. Acesso em 14 fev. 2021.

8. BRASIL, Ministério da saúde. Agência Nacional de Vigilância Sanitária; Formulário de Fitoterápica $2^{a}$ edição Farmacopéia Brasileira - Brasília 2021.pág. 64. Disponível em: https://www.gov.br/anvisa/pt-br/assuntos/farmacopeia/formulario-fitoterapico/arquivos/2021fffb2-final-c-capa2.pdf. Acesso em: 19 jun. 2021.

9. Governo do Distrito Federal. Secretaria de Estado de Saúde Subsecretaria de Atenção Integral à Saúde. Coordenação de Atenção Especializada à Saúde. Diretoria de Assistência Farmacêutica Gerência do Componente Básico da Assistência Farmacêutica. Núcleo de Farmácia Viva (Nufarv)- 2018. Disponível em: https://www.saude.df.gov.br/wpconteudo/uploads/2020/06/Guia-Fitoter\%C3\%A1 picos-Oficinais-do-N\%C3\%BAcleo-deFarm\%C3\%A1cia-Viva-8\%C2\%AA-edi\%C3\%A7\%C3\%A3o-2018.pdf. Acesso em: 10 ago. 2021.

10. SERVIN, S. C. N. et al; Protocolo de acolhimento com classificação de risco sistema de saúde (SUS) HOSPITAIS MUNICIPAIS/ SÃO LUÍS/MA. Disponível em https://bvsms.saude.gov.br/bvs/publicacoes/protocolo_acolhimento_classificacao_risco.pdf. Acesso em: 18 mar. 2021.

11. BRASIL, Lei nº 13.709. Lei Geral de Proteção de Dados Pessoais (LGPD). Diário Oficial da União, Brasília (DF). 15 de Agosto de 2018, p.59. Disponível em: https://www.planalto.gov.br/ccivil_03/_ato2015-2018/2018/lei/113709.htm 
12. PRADO, M. A. S. dos A.; MATSUOK, J. T.; GIOTTO, A. C. Importância das Farmácias Vivas no âmbito da produção dos medicamentos fitoterápicos. Revista de Iniciação Científica e Extensão, $[S . \quad$ l.], $\quad$ v. $\quad 1, \quad$ n. $\quad 1, \quad$ p. $\quad 32-37, \quad 2018 . \quad$ Disponível em: https://revistasfacesa.senaaires.com.br/index.php/iniciacao-cientifica/article/view/45.Acesso em: 8 jul. 2021.

13. GUIMARAES, L. da S; CRUZ, M. C da. Exercícios terapêuticos: a cinesioterapia como importante recurso da fisioterapia. Revista Lato \& Sensu, Belém, v. 4, n. 1, p. 3-5, out, 2003. Disponível em: http://fisio-tb.unisul.br/arquivos/artigo_Cinesioterapia.pdf. Acesso em: 19 jul. 2021.

14. SANT’ANA, H. G. F.; ROCHA MARIA, R. de A.; DE LIMA, V. A. S.; NEVES, R. H. S. Modalidades terapêuticas no tratamento e prevenção da dor muscular tardia- revisão de literatura. Caderno de Graduação - Ciências Biológicas e da Saúde - UNIT - ALAGOAS, [S. l.], v. 4, n. 1, p. 147, 2017. Disponível em: https://periodicos.set.edu.br/fitsbiosaude/article/view/3935. Acesso em: 19 jul. 2021. 15. NOGUEIRA, A. F..; COSTA, B. H. da; ARRUDA, E. F. de; LEITE, M. B.; SOUSA, C. S. de. Principais distúrbios traumato-ortopédicos atendidos em clínicas-escola de fisioterapia. Revista Científica da Faculdade de Educação e Meio Ambiente, [S. l.], v. 7, n. 2, p. 33-44, 2016. DOI: 10.31072/rcf.v7i2.395. Disponível em: http://www.faema.edu.br/revistas/index.php/Revista-FAEMA/article/view/395. Acesso em: 19 jul. 2021.

16. NASCIMENTO, H. B. do, et al .Principais patologias e recursos fisioterapêuticos utilizados na fisioterapia traumato-ortopédica. Anais da XVIII Mostra Acadêmica do Curso de $\begin{array}{llllll}\text { Fisioterapia, } & \text { v. } & 8, & \text { n.1, } & 2020 . & \text { Disponível }\end{array}$ http://anais.unievangelica.edu.br/index.php/fisio/article/view/5667/3125. Acesso em: 19 jul. 2021. 
17. PIMENTA C.A.M, TEIXEIRA M.J. Dor no idoso. In: Duarte YAO, Diogo MJE. Atendimento domiciliar um enfoque gerontológico. São Paulo: Atheneu; 2000. p.373-87.

18. DEllarozA M. S. G; FURUYA, R. K.; CABRERA, M. A. S; MATSUO, T. ;TRELHA, C. ; YAMADA, K. N., PACOLA L. Caracterização da dor crônica e métodos analgésicos utilizados por idosos da comunidade. Rev Assoc Med Bras 2008; 54(1): 36-41. Disponível

em: https://www.scielo.br/j/ramb/a/d37LZLZ49k66xjWdjn7nG5v/?lang=pt\&format=pdf. Acesso em: 20 jul. 2021.

19. MARQUEZ, J. O. A dor e os seus aspectos multidimensionais. Cienc. Culto., São Paulo, v. 63, n. 2, pág. 28-32, abril de 2011. Disponível em http://cienciaecultura.bvs.br/scielo.php?script=sci_arttext\&pid=S0009-

67252011000200010\&lng=en\&nrm=iso. Acesso em: 21 jun. 2021. http://dx.doi.org/10.21800/S0009-67252011000200010.

20. CALIL A. M, PIMENTA C. A.Gravity of injury and analgesia in patients who suffered traffic accidents. Acta Paulista de Enfermagem [online]. 2008, v. 21, n. 3, pp. 398-403. Disponível em: https://doi.org/10.1590/S0103-21002008000300003. Epub 09 Oct 2008. ISSN 1982-0194. Acesso em: 21 jul. 2021

https://doi.org/10.1590/S0103-21002008000300003.

21. PEREIRA L.V, SOUSA F.A. Psychophysical evaluation of the descriptors of pain in the postoperative. Revista Latino-Americana de Enfermagem [online]. 2007, v. 15, n. 3 , pp. 474479. Disponível em: https://doi.org/10.1590/S0104-11692007000300017. ISSN 1518-8345. https://doi.org/10.1590/S0104-11692007000300017.

22. BIOMEDICINA PADRÃO. CÂMARA. B. As 5 fases da inflamação e os 5 sinais cardinais. Sexta-Feira, 03 de maio de 2013. Disponível em: 
https://www.biomedicinapadrao.com.br/2013/05/as-5-fases-da-inflamacao-e-os-5-sinais.html Acesso em: 22 de jul. 2021.

23. AGNE J.E. Eletrotermoterapia: teoria e prática. Santa Maria: Orium, 2005.p.221-227.

24. CAMERON M. H. Agentes físicos na reabilitação - da pesquisa à prática.3.ed. Rio de Janeiro: Elsevier, 2009.p. 131-176. 